\title{
A GRASP ALGORITHM FOR THE CONTAINER LOADING PROBLEM WITH MULTI-DROP CONSTRAINTS*
}

\author{
D. Alvarez Martínez ${ }^{1}$, R. Alvarez-Valdes ${ }^{2 * *}$ and F. Parreño ${ }^{3}$
}

Received March 13, 2015 / Accepted March 31, 2015

\begin{abstract}
This paper studies a variant of the container loading problem in which to the classical geometric constraints of packing problems we add other conditions appearing in practical problems, the multidrop constraints. When adding multi-drop constraints, we demand that the relevant boxes must be available, without rearranging others, when each drop-off point is reached. We present first a review of the different types of multi-drop constraints that appear in literature. Then we propose a GRASP algorithm that solves the different types of multi-drop constraints and also includes other types of realistic constraints such as full support of the boxes and load bearing strength. The computational results validate the proposed algorithm, which outperforms the existing procedures dealing with multi-drop conditions and is also able to obtain good results for more standard versions of the container loading problem.
\end{abstract}

Keywords: container loading, multi-drop, load-bearing strength, heuristics, GRASP.

\section{INTRODUCTION}

The single container loading problem (CLP) is one of the most challenging problems in cutting and packing. It is a three-dimensional optimization problem in which we have to pack a set of rectangular objects, boxes, into a large rectangular object, container, in such a way that the packing optimizes some criterion while satisfying a set of constraints. It is a critical part of any supply chain because it has to be solved on a daily basis in many different situations, varying in the type and characteristics of the goods to be transported, the number and types of trucks or containers to be used, and the specific loading constraints of each company. Loading these containers efficiently, that is, minimizing the empty spaces inside them, is not only an economic necessity, but also an ecological issue due to the adverse impact of increased traffic on environmental resources.

\footnotetext{
*Invited paper.

**Corresponding author.

${ }^{1}$ La Salle University, Faculty of Engineering, Industrial Engineering, Bogota, Colombia. E-mail: akavallo@gmail.com

${ }^{2}$ University of Valencia, Department of Statistics and O.R., Doctor Moliner, 50, 46100 Burjassot, Valencia, Spain.

E-mail: ramon.alvarez@uv.es

${ }^{3}$ University of Castilla-La Mancha, Department of Mathematics, Albacete, Spain. E-mail: francisco.parreno@uclm.es
} 
It is therefore not surprising that many solution approaches have appeared in the scientific literature in the last twenty years. Nevertheless, most of the academic research has focused on the basic problem in which the volume occupied has to be maximized, subject to geometric constraints that prevent the boxes from overlapping each other and exceeding the dimensions of the container. As long ago as 1995, Bischoff \& Ratcliff [5] warned us that a number of factors, which are frequently of importance in practical situations, had not received sufficient attention in the OR literature. They listed twelve practical conditions that have to be considered when solving practical problems for which feasible loading plans have to be constructed. Since then, there has been a clear line of research in which these practical conditions have been added to the basic geometric problem. More recently, Bortfeldt \& Wäscher [9] have written an exhaustive review of the container loading problem and its relevant practical constraints.

In this paper, we focus our attention on one set of these realistic constraints, the multi-drop constraints. In some situations the loading plan has to take into account the fact that the boxes must be delivered to different places. Every time a drop-off point is reached, the relevant boxes must be available to be unloaded without rearranging other boxes. This condition adds additional constraints on the order and the position in which the boxes are placed in the container. Depending on the way the boxes are unloaded and depending on the requirements of the company, we may have different multi-drop constraints.

The paper is organized as follows. In Section 2 we review the existing heuristic approaches to the basic container loading problem, and discuss and classify the additional constraints that have been proposed and included in the published procedures. The multi-drop constraints are described in more detail in Section 3, reviewing and discussing the different ways in which they have been used by different authors. The main objective of this study is to develop an algorithm that can accommodate the different variants of multi-drop constraints. Section 4 describes the problem being solved, Section 5 contains the constructive and GRASP algorithms, and Section 6 describes the computational results, which show that our algorithm is able to outperform all the published approaches. Finally, some conclusions are drawn.

\section{ADDING REALISTIC CONSTRAINTS TO THE BASIC CONTAINER LOADING PROBLEM}

The basic Container Loading Problem can be defined as the problem of placing a set of boxes into the container respecting the geometric constraints: the boxes cannot overlap and cannot exceed the dimensions of the container. According to the improved typology for cutting and packing problems proposed by Wäscher et al. [48], the container loading problem can be classified as a three-dimensional rectangular single large object placement problem (3D-SLOPP) if the set of boxes is weakly heterogeneous, or a single knapsack problem (3D-SKP) if the set of boxes is strongly heterogeneous. In any case, the CLP is an NP-hard problem because the NP-hard one-dimensional knapsack problem can be transformed into it. As a consequence, few exact algorithms have been proposed to solve the problem (Fekete et al. [18], Martello et al. [35], Junqueira et al. [31]). In contrast, there are many heuristic and metaheuristic procedures based 
on different approaches to packing boxes into the container. Pisinger [43] and Fanslau \& Bortfeldt [17] have classified these approaches as follows:

\section{Wall-building approach}

The wall-building approach, introduced by George \& Robinson [23], fills the container with a number of vertical layers (walls) across the length of the container. It has been used by Bortfeldt \& Gehring [7] in their Genetic Algorithm, by Pisinger [43] in his tree-search method, and by Moura \& Oliveira [38] in their GRASP algorithm.

\section{Stack-building approach}

The stack-building approach, proposed by Gilmore \& Gomory [24], packs the boxes into stacks which are arranged on the floor of the container by solving a two-dimensional packing problem. This method is used by Bischoff \& Ratcliff [5] in their greedy algorithm, in the Genetic Algorithms by Bortfeldt \& Gehring [7], and Gehring \& Bortfeldt [20], and by Hifi [26] in his tree-search method.

\section{Horizontal layer building approach}

The container is filled with horizontal layers from bottom to top, trying to cover the maximum surface of the container. This is the approach of Bischoff \& Ratcliff [5] in their greedy algorithm and of Terno et al. [47] in their branch and bound procedure.

\section{Block-building approach}

The container is filled with blocks, usually composed of boxes of the same type, although some authors consider blocks that combine boxes of different types. Many authors have used this approach in a number of different procedures. Some examples are the Tabu Search algorithm by Bortfeldt et al. [8], the tree-search by Eley [15], the hybrid Simulated Annealing/Tabu Search by Mack et al. [34], the GRASP algorithm by Parreño et al. [40], and the hybrid GRASP/VNS by Parreño et al. [42].

\section{Guillotine cutting approach}

This approach is based on a slicing tree representation of a packing plan. The branches correspond to the guillotine partitioning of container regions into smaller parts, where the leaf nodes correspond to the boxes. The graph-search method by Morabito \& Arenales [37] is based on this approach.

Nevertheless, in order to obtain packing plans that are useful in real problems, besides the geometric conditions many other constraints have to be taken into account. These realistic constraints have been added to the basic problem by many authors in recent years, though much more work is still needed. Following the survey by Bortfeldt \& Wäscher [9], we review and classify here the most important contributions that have appeared in the literature. The multi-drop constraints that are the object of this study will be fully described in Section 3. 


\subsection{Container constraints}

Global container constraints refer to the weight limit and weight distribution inside the container. The weight limit is the maximum weight that can be loaded into the container. When the cargo is heavy, weight becomes a very restrictive condition, more so than the volume occupied by the boxes. Gehring \& Bortfeldt [20], Bortfeldt et al. [8], Terno et al. [47], and Egeblad et al. [14] are some of the authors who address this weight limit constraint.

Weight distribution constraints require the weight of the cargo to be spread across the container floor. An unbalanced container can produce problems when lifted by a crane. Also, when transported by truck, the cargo distribution has to take into account the maximum weight supported by the truck axles. One way of achieving a good balance is to require that the center of gravity of the load must lie in the geometric center of the container, possibly with some tolerances, as in the studies by Bischoff \& Marriott [4], Gehring \& Bortfeldt [19], and Bortfeldt \& Gehring [7].

\subsection{Item constraints}

Constraints directly related to items are priority constraints, orientation, and load bearing constraints. When the container is not large enough to accommodate all the items, a decision about which items to load has to be taken. Sometimes this is done by using priorities, related to delivery deadlines or to the shelf life of the products, as in Bischoff \& Ratcliff [5] and Ren et al. [45].

One of the most common constraints is the orientation constraint, which forbids some of the six potential orientations of a box. It usually limits the vertical orientation to one dimension ("This way up!"), allowing $90^{\circ}$ rotations of items on the horizontal plane, as in Haessler \& Talbot [25], and Iori \& Martello [28]. Nevertheless, sometimes only one orientation is allowed and boxes cannot be rotated, as in Morabito \& Arenales [37] and Junqueira et al. [31]. Conversely, in some cases all six possible orientations are permitted and boxes can rotate freely, as in Parreño et al. [40] and Ratcliff \& Bischoff [44].

Load bearing constraints limit the way in which boxes can be placed on top of each other. It is usual that the boxes to be packed differ in weight and also in their ability to withstand pressure from the weight resting on them. This constraint can limit the number of boxes a box can bear above it, as in Bischoff \& Ratcliff [5] or can prohibit a particular type of product being placed on top of another type, as in Terno et al. [47]. Nevertheless, the most usual load bearing constraint limits the weight which can be applied on top of every box. Some recent examples are the papers by Bischoff [3], Christensen \& Rousoe [12], Junqueira et al. [31], and Alonso et al. [1].

\subsection{Cargo constraints}

When all the boxes cannot be accommodated in the container, apart from the decision about the loading of each box there can be other constraints, such as the complete-shipment constraints, requiring all the components of a product that have to be assembled at destination to be loaded together, as in Bischoff \& Ratcliff [5], Iori \& Martello [28], and Moura \& Oliveira [38]. 
Positioning constraints restrict the allocations of the item in the container. They can refer to absolute positions (some boxes near the container door), as in Haessler \& Talbot [25], Bortfeldt $\&$ Gehring [7], and Terno et al. [47], or to relative positions (certain items have to be placed together or at a certain distance from each other) as in Bischoff \& Ratcliff [5].

\subsection{Load constraints}

This type of constraint is related to the properties of the final arrangement of the items in the container. The most important of these properties is the stability of the cargo. Two types of stability can be distinguished, vertical and horizontal stability.

Vertical or static stability prevents items from falling. A box must be supported from below by other boxes to a given percentage. If this percentage is $100 \%$, we speak of full support (Bortfeldt \& Gehring [7], Araujo \& Armentano [2], Fanslau \& Bortfeldt [17], Ngoi et al. [39]). Lower percentages correspond to partial support (Jin et al. [29], Junqueira et al. [31]).

Horizontal or dynamic stability ensures that the boxes cannot move while the container is being moved. Full horizontal stability means that each box is either adjacent to other boxes or to the container wall on all its sides ([29], [31]). Bischoff \& Ratcliff [5] and Eley [15] evaluate the lateral support of the load by the percentage of boxes that are not in contact with other boxes or a container wall.

\section{MULTI-DROP CONSTRAINTS}

The first authors who considered multi-drop situations were Bischoff \& Ratcliff [5]. According to them, if a container is to carry consignments for a number of different destinations, it is desirable not only to place items within the same consignment close together, but also to order the consignments within the container so as to avoid, as far as possible, having to unload and reload a large part of the cargo several times. More recent studies are usually stricter, imposing the condition that the arrangement of the boxes within the container should reflect the sequence in which they have to be delivered, in order to avoid any unloading and reloading operations. In their survey, Bortfeldt \& Wäscher [9] consider this constraint as a combination of absolute and relative positioning constraints, so it could have been included in Section 2.3.

The literature that includes multi-drop constraints can be divided into two main groups. The first group consists of studies that address packing problems in which multi-drop constraints are considered. The second group includes studies that address combined problems of routing and packing.

In the first category, Bischoff \& Ratcliff [5] propose the first procedure for taking multi-drop constraints into account, which involves loading a single customer at a time. Lai et al. [32] transform a similar problem into a graph and develop methods to reduce the graph and to find the maximal cliques in the graph. Terno et al. [47] develop an algorithm for the multi-pallet loading problem, taking into account multi-drop and connectivity constraints. Jin et al. [29] use simulated 
annealing and design their algorithm to solve the problem with fixed loading (unloading) orders. None of the previous algorithms has explicit results for multi-drop constraints. Christensen \& Rousoe [12] present the first results of an algorithm dealing with multi-drop and load-bearing constraints. They propose a heuristic based on a tree search framework, using maximal spaces. Liu et al. [33] propose an algorithm for a case of multi-drop in which each box has a different destination. Their algorithm uses a scheme in which the next space to pack a box is randomly chosen among the list of empty spaces. Junqueira et al. [30] develop an exact model to solve small instances of a container loading problem with multi-drop constraints. Their approach is based on a mixed integer linear programming model. The results indicate that they are able to handle only problems of a moderate size. More recently, Ceschia \& Schaerf [10] have proposed a metaheuristic algorithm for multi and single container problems. Their local search procedure works on the space of sequences of boxes to be loaded, while the actual load is obtained by invoking, at each iteration, a specialized procedure. This procedure inserts the boxes into the container using a deterministic heuristic which produces a load that is feasible according to the constraints.

The second approach dealing with multi-drop constraints combines routing and packing. Gendreau et al. [21] propose an algorithm to solve the combined problem with three-dimensional packing constraints. This problem is known as the three-dimensional loading capacitated VRP (3L-CVRP). Other approaches have reduced the loading constraints, so that only one- or twodimensional loads are considered. The two-dimensional loading capacitated VRP (2L-CVRP) is addressed by Gendreau et al. [22] and Iori et al. [27]. The one-dimensional variant is considered by Doerner et al. [13]. Common to these approaches is that the two problems are solved separately. A vehicle routing algorithm suggests routes that are checked for feasibility by using a packing algorithm. For this setup to work in a practical setting, solutions to the CLP must be obtained fast, as typically many routes need to be checked. Furthermore, when the CLP is combined with vehicle routing, the multi-drop constraints on CLP become essential. Iori \& Martello [28] have produced a review of this type of problem. In recent years there have been several studies developing efficient algorithms. Zhu et al. [49] develop a two-stage tabu search for the 3L-CVRP and for the M3L-CVRP, the case in which the loading is done manually. Bortfeldt [6] propose a very efficient algorithm including a tabu search algorithm for routing and a tree search algorithm for loading. Ceschia \& Schaerf [10] propose an extension of 3L-CVRP introduced by Gendreau et al. [21]. They include additional constraints in relation to the stability of the cargo, the fragility of items, and the loading and unloading policy, and consider the possibility of split deliveries, so that each customer can be visited more than once.

When reviewing the above papers, it can be seen that the condition of avoiding reloading operations when unloading the boxes at each drop-off point can be implemented in practice in different ways, depending on whether the container has only one unloading door or more than one, and on the machinery that is available to do these operations: whether it is done manually or using a forklift. Therefore, when defining which items can be unloaded at a destination while respecting the multi-drop constraints, we can distinguish three main types: 


\section{- Boxes that are visible}

There has to be a passage from the box to an entrance of the container, that is, the boxes to be unloaded at a destination have to be completely visible from one or more sides of the container in the destination point, or be blocked only by boxes for the same destination. In most of the papers this condition has to be satisfied from the unloading door and from the top of the container. Several authors use this definition, for instance Gendreau et al. [21], Christensen \& Rousoe [12], and Ceschia \& Schaerf [10]. When a customer is visited, none of the corresponding boxes may be stacked beneath nor be blocked by boxes for customers that are to be visited later. This constraint is violated if for any pair of boxes $i$ and $j$ such that $i$ has to be unloaded before than $j, j$ is on top of $i$ (i.e. the base of $j$ is higher than the top of $i$ and they overlap horizontally), or $j$ is in front of $i$ (i.e. the back of $j$ is in front of the front of $i$, and they overlap across the container). This case reflects the unloading of boxes by forklifts, where they are first elevated and then moved towards the unloading door, as can be seen in Figure 1, in which the boxes for the next costumer appear in light grey color.

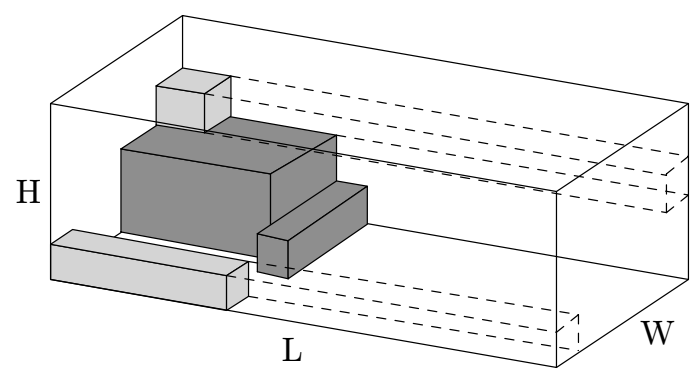

$\mathrm{L}$

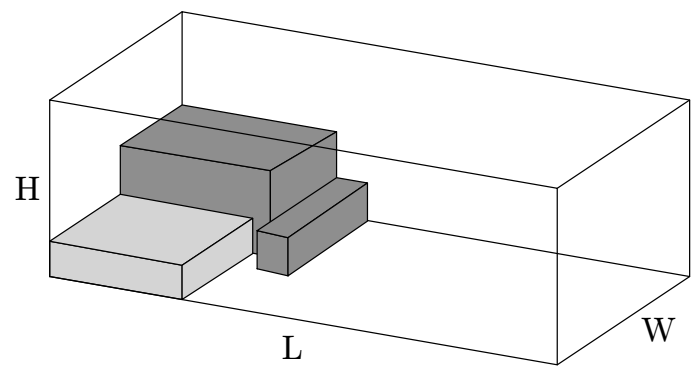

(a) Visible

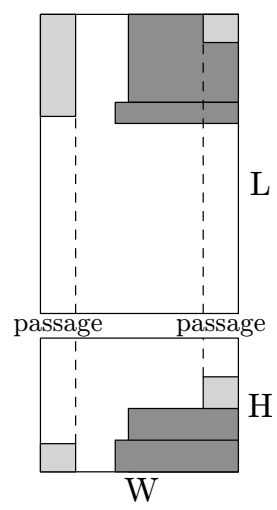

W 


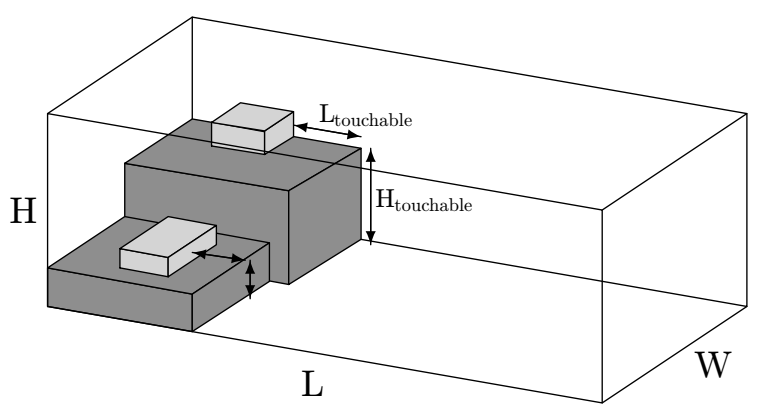

$\mathrm{L}$

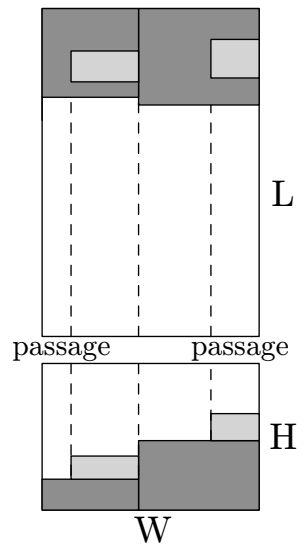

(a) Visible and touchable
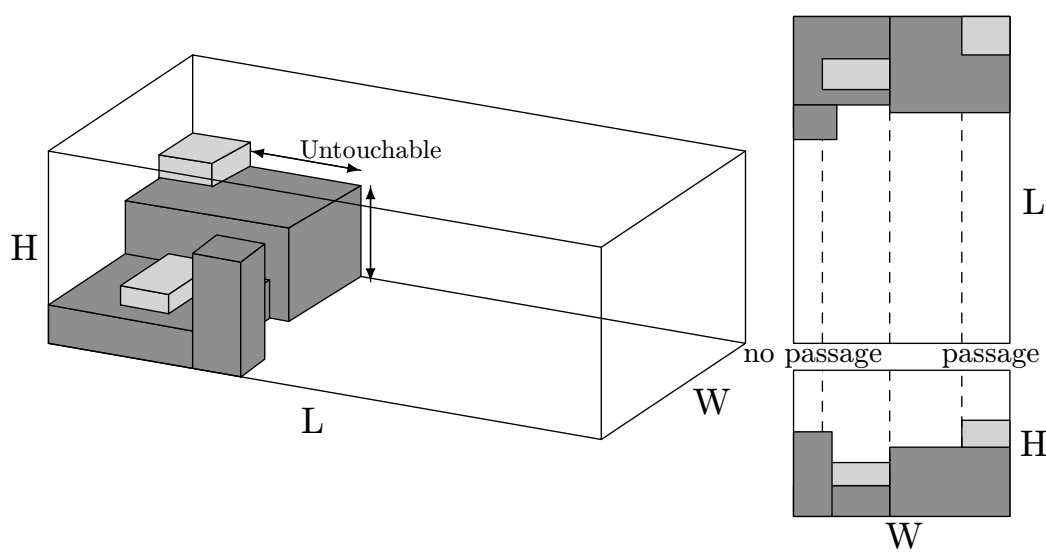

(b) Not visible or not touchable

Figure 2 - Applying the visible and touchable criterion.

\section{- Boxes that are touchable}

This condition imposes a further constraint apart from visibility. We consider that although a box may be visible, in some circumstances it could be very difficult to reach for a person or for a machine. Imagine that we have a large narrow corridor inside the container that makes a box visible, such as that on the top level in Figure 1(a). Maybe we can use this corridor to reach and unload this box, but in some cases it is not physically possible to do so. Then, Liu et al. [33] define reachability in terms of the position of each box in relation to the partial packing existing in the container when it arrives at the destination at which the box has to be unloaded. A box is considered reachable if it can be reached by a worker or forklift of given dimensions. In other words, the box is considered reachable if the distance between it and the nearest position in which a human operator or a forklift can be located is less than or equal to a fixed quantity. Liu et al. [33] define two measures. 
For the delivery person to be able to touch item $b_{i}$ with his/her hands, $L_{\text {touchable }}$ and $H_{\text {touchable }}$ have to satisfy the following constraints:

$$
\begin{gathered}
L_{\text {touchable }}+H_{\text {touchable }} \leq 200 \\
L_{\text {touchable }} \leq \min \left\{200-z_{i}, 60\right\}
\end{gathered}
$$

where $z_{i}$ is the height at which the box is placed (see Fig. 2). Ceschia et al. [11] use only one parameter, the horizontal distance. $L_{\text {touchable }}$ is the distance along the axis $L$ from the box to the nearest place we can use to reach the box.

\section{- Boxes that are separated from those of other customers}

Junqueira et al. [30] define reachability as a function of a parameter $\delta$, which indicates how many units of length beyond the "border" between boxes of consecutive destinations the worker is allowed to go in order to unload the boxes. They define the "border" as a virtual wall defined after all boxes for a destination have been packed inside the container. These walls separate the boxes for each customer across the length of the container. If this parameter $\delta=0$ (Fig. 3), boxes for the next customer (in light gray) must be completely separated from those for the previous customer (in dark gray). The worker is not allowed to cross this virtual wall. In the general case, the worker is allowed to go only partially beyond the virtual wall. In Figure 4, with $\delta=l_{i}$, light gray boxes can be placed beyond the wall defined by dark gray boxes, but one of their sides must always be on the virtual wall.
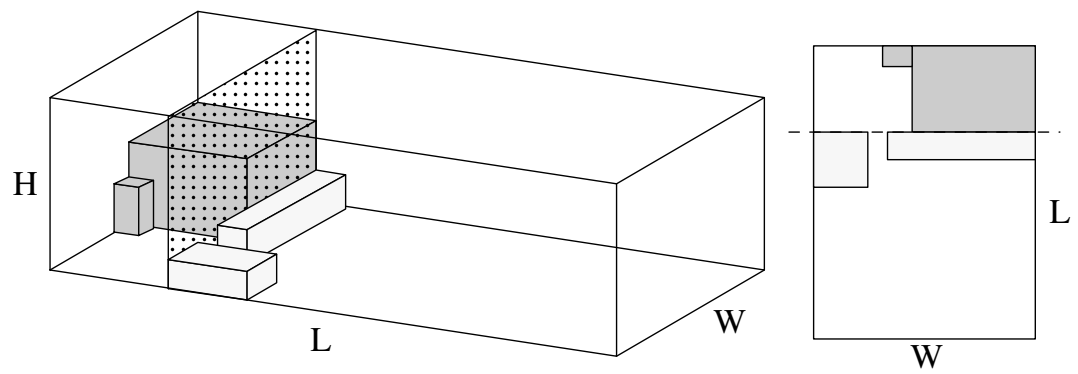

(a) Feasible with $\delta=0$
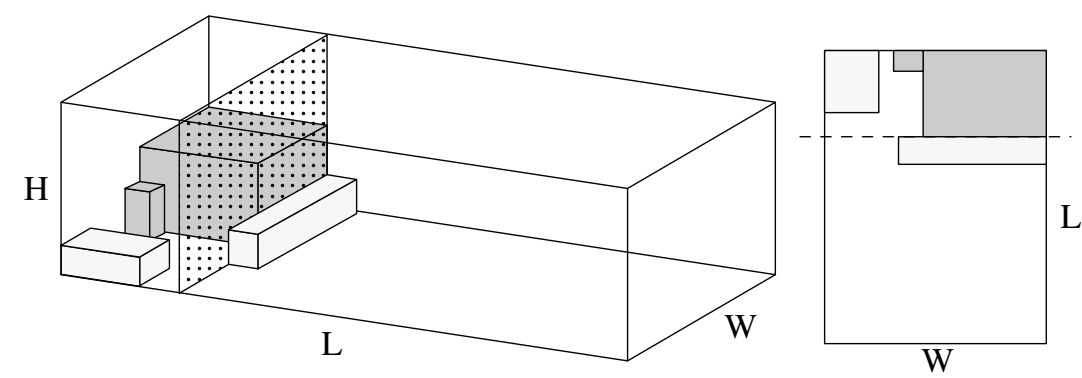

(b) Unfeasible with $\delta=0$

Figure 3 - Applying the Junqueira et al. virtual walls with $\delta=0$. 


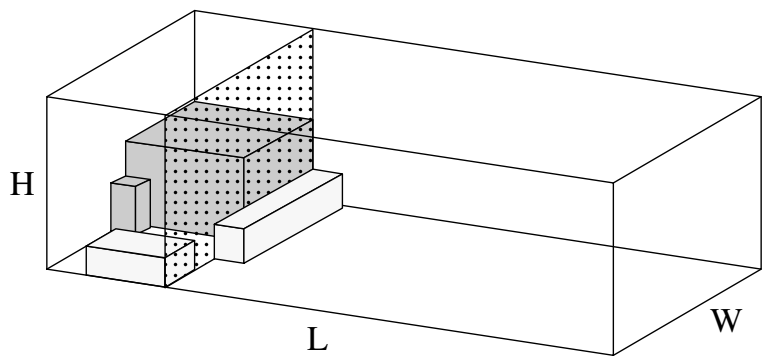

(a) Feasible with $\delta=l_{i}$
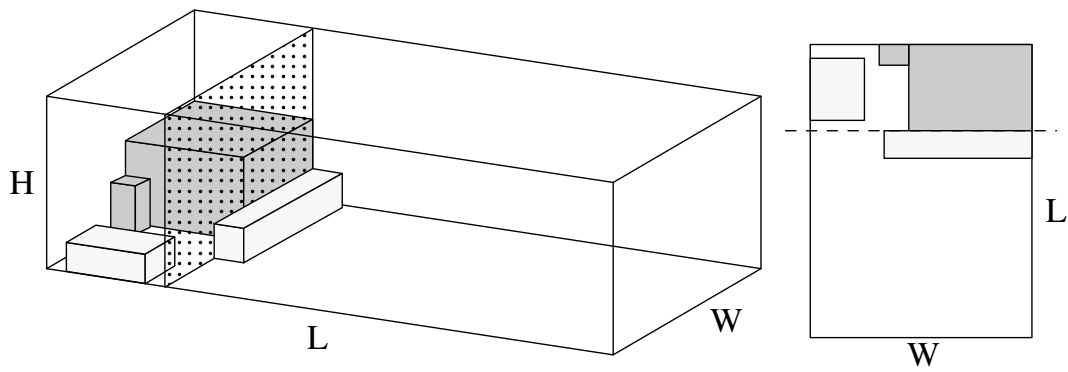

(b) Unfeasible with $\delta=l_{i}$

Figure 4 - Applying the Junqueira et al. virtual walls with $\delta=l_{i}$.

In these problems in which we have several clients, there is another question to be addressed: can we load boxes for client $j$ before all the boxes for a previous client $i$ have been packed? We can have two cases:

\section{- Restricted}

We have to pack the complete shipment for each customer in the given order. That is, it is not possible to pack some boxes for customer $j$ unless all the boxes for customer $i$ have been packed $(j>i)$. This is the most common circumstance when packing with multi-drop constraints (Christensen \& Rousoe [12], Ceschia \& Schaerf [10]).

\section{- Unrestricted}

In this case, it is possible to pack boxes for customer $j$ even though there are some boxes for customer $i$ which have been left unpacked $(j>i)$. In this case these boxes would be left out of the loading. In Liu et al. [33] and in Junqueira et al. [30], the objective is to maximize the total volume packed, and this can be achieved by leaving out boxes for several customers.

In Figure 5 we have three clients with boxes in different shades of gray (Fig. 5(a)) and we have a two-dimensional container of dimensions $(6,2)$. Considering that the objective is to maximize the volume packed, in Figure 5(b) we can see the optimal solution for the restricted case, with 
value 10, while in Figure 5(c) we see the optimal solution packing all the boxes for clients 1 and 3 , with value 11, but leaving out the intermediate client 2. Finally, in Figure 5(d) we can see the optimal solution for the less constrained case, with value 12 , packing some, but not all, of the boxes for the three clients.
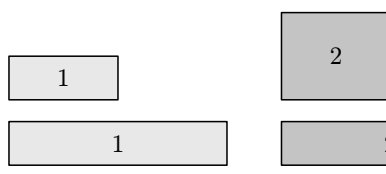

2

(a) Pieces

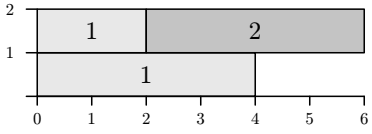

(b) Restricted

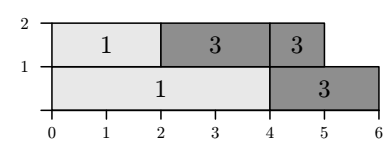

(c) Unrestricted I

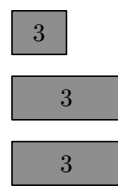

(d) Unrestricted II

Figure 5 - Packing different subsets of clients' boxes.

\section{PROBLEM DESCRIPTION}

We have a container of dimensions $(L, W, H)$ that has to be filled with a set of $n$ boxes, $B_{i}, i=$ $1, \ldots, n$. Each box has dimensions $\left(l_{i}, w_{i}, h_{i}\right)$ in $\mathrm{cm}$. and weight $w_{i}$ in $\mathrm{kg}$. and a destination or drop number $d_{i}$. The objective is to find an orthogonal packing of the boxes so as to maximize the container volume utilization, subject to these constraints:

\section{- Full support:}

The base of each box has to be placed on the floor of the container or completely on top of other boxes.

\section{- Allowed orientation:}

Each box has a set of allowed orientations due to its content or its structure. The orientations are denoted by $o_{i j}$, where $i=1, \ldots, n$ represents the box and $j=1,2,3$ the orientation, that is, the dimension of the box that can be placed upright: $o_{i 1}=1$ if dimension $l$ of box $i$ can be upright and 0 if it may not, $o_{i 2}=1$ if dimension $w$ can be upright and 0 if it may not, and $o_{i 3}=1$ if dimension $h$ can be upright and 0 if it may not. At least one of these parameters must be 1 for each box $i$.

- Load-bearing strength:

Each box placed in one allowed orientation can bear a maximum weight $m_{i j}$ on top of it, expressed in $\mathrm{gr} / \mathrm{cm}^{2}$, where $i=1, \ldots, n$ represents the box and $j=1,2,3$ the orientation. As Bischoff [3] points out, there are several ways in which the weight of a box is 
transmitted downwards onto the boxes supporting it from below. In practice, it depends on the stiffness of the supporting surface: the more rigid it is, the more the weight is spread over the whole surface. When using soft materials like cardboard, the weight of the box above is essentially transmitted down onto the contact area only. We will assume this latter situation and therefore when a box $k$ is put on top of another box $i$, its weight $w_{k}$ will be divided by its base area to calculate the pressure, $p_{k}$ in $\mathrm{gr} / \mathrm{cm}^{2}$, exerted on the supporting box. If this pressure $p_{k}$ exceeds the load-bearing capacity of the box below, $m_{i j}$, the packing is not feasible. Otherwise, the remaining load-bearing capacity of box $i$ will be reduced by $p_{k}$.

\section{- Multi-drop:}

We admit the three possible definitions of multi-drop constraints described in the previous section. We have designed an algorithm able to deal with all kinds of multi-drop constraints. Depending on the type of constraint being considered, some steps of the algorithm will be changed, as will be explained in the next section.

\section{A GRASP ALGORITHM}

The GRASP algorithm was developed by Feo \& Resende [16] to solve hard combinatorial problems. For an updated introduction, see Resende \& Ribeiro [46]. GRASP is an iterative procedure combining a constructive phase and an improvement phase. In the constructive phase, a solution is built step by step, adding elements to a partial solution. The constructive phase is iterative, greedy, randomized, and adaptive. In the next subsections we describe a constructive procedure, a randomization strategy which will be embedded in the constructive process, some movements for the improvement phase, and a diversification phase which can be included in the iterative structure.

\subsection{Constructive Algorithm}

The basis of our constructive algorithm is the constructive procedure proposed by Parreño et al. [41] for the container loading problem. The algorithm works on maximal spaces. As the selected box is packed into a new space, three new maximal spaces are created. The constructive algorithm uses an updated list of maximal spaces and a list of boxes for the current client still to be packed. The most difficult part in this case is the management of the empty spaces when all the boxes for a client have been packed. In this case, before starting to pack the boxes for the next client, some spaces have to be removed from the list and others have to be updated in order to satisfy the multi-drop constraints. The details of the algorithm can be found in [41]. For completeness we will outline its main steps here.

\section{Step 0: Initialization}

$S=$ the list of empty maximal spaces created when packing the boxes. Initially, $S$ is just the empty container.

$\mathcal{B}=\left\{B_{1}, B_{2}, \ldots, B_{n}\right\}$, the set of boxes still to be packed, ordered by customer. 


\section{Step 1: Choosing the maximal space from $S$}

We have a list $S$ of empty maximal spaces, which are the largest empty parallelepipeds available to be filled with boxes. We use two alternative strategies to choose the maximal space. We alternate between choosing the maximal space with the minimum distance to the back of the container and choosing the maximal space with the largest coordinate $z$. The reason behind these strategies is to fill the back of the container first or to pile boxes forming stacks.

\section{Step 2: Choosing the boxes to pack}

Once a maximal space $S$ has been chosen, we consider the remaining boxes of the current customer fitting into $S$ in order to choose which one to pack. If there are several boxes of the same dimensions, we consider the possibility of packing a block, that is, packing several of these boxes arranged in a rectangular array with several rows and columns.

Two criteria have been considered to select the configuration of boxes:

\section{Best-Volume:}

The box or block producing the largest increase in the volume occupied by boxes.

\section{Best-Fit:}

The box or block which best fits into the maximal space. We compute the distance from each side of the box or block to each side of the maximal space and put these distances in a vector in non-decreasing order. The box or block is chosen using the lexicographical order.

If we are using the visibility criterion, the corner into which the box or the block is packed is the corner of the maximal space with the shortest distance to the origin of the container. In the other two cases, we have to check how and where we put the box or the block in order to make it reachable, always trying to put it as far back as possible so as to maximize the remaining usable space.

\section{Step 3: Updating the list $S$ for the current customer}

Unless the box or block fits exactly into space $S$, packing it produces new empty maximal spaces which will replace $S$ in the list $S$. Moreover, as the maximal spaces are not disjoint, the box or block being packed can intersect with other maximal spaces which will have to be reduced.

Once the new spaces have been added and some of the existing ones modified, we check the list and eliminate possible inclusions. The list $\mathcal{B}$ is also updated and the maximal spaces that cannot accommodate any of the boxes still to be packed are eliminated from $S$. If $S=\emptyset$ or $\mathcal{B}=\emptyset$, the procedure ends. Otherwise, if we have not finished packing the boxes for the current customer, we go back to Step 1 .

\section{Step 4. Updating the list $S$ for a new customer}

We have to update the list of maximal spaces for the next customer. This depends on the type of multi-drop constraint: 
- Visible: We have to remove from the list all the maximal spaces that are not completely visible from the front of the container. We update the maximal spaces that have some part that is visible and other non-visible.

- Touchable and visible: Apart from the spaces removed when applying the previous criterion, we have to remove or update all the spaces that are not touchable.

- Separated: If we work with virtual walls separating the customers and a given value of $\delta$, we have to remove or update all the maximal spaces whose $x$-coordinate is lower than the position of the virtual wall minus $\delta$.

Figure 6 shows an example of the different ways of updating the maximal spaces when the boxes for a client have been packed. The left part of the figure shows the view from the top and the right part the view from one side. Boxes 1 to 5 , corresponding to the first customer, have been packed. Using the first criterion, visibility, the space on the floor behind piece 4 cannot be used, and neither can the space on top of piece 1, because piece 3 is taller. Using the second criterion, touchability, the space above piece 3 cannot be reached. Also, as piece 2 is very high, only part of the space above it is touchable. The third criterion, separation with $\delta=0$, builds a virtual wall at the right-hand side of the last piece and this wall cannot be penetrated to pack the next customer's boxes.

As in Parreño et al. [41], two criteria have been considered to select the configuration of boxes:

- The block of boxes producing the largest increase in the objective function. This is a greedy criterion in which the space is filled with the block producing the largest increase in the volume occupied by boxes.

- The block of boxes which fits best into the maximal space. We compute the distance from each side of the block to each side of the maximal space, order these distances in a vector in lexicographical order, and select the block with the minimum distance.

\subsection{Randomization}

For each type of box and each allowed orientation, a block is built, according to the criterion selected. From the set of all possible blocks, a restricted candidate list $(R C L)$ is built and the block to be packed is randomly selected from this list. We have used a value-based Restricted Candidate List: the candidates are selected according to their quality. If the value of the candidate's objective function is better than a threshold, the candidate is placed on the list. In the course of the process of building blocks we get $C_{\min }$ and $C_{\max }$, the largest and the smallest values of all the candidates, and the value of each candidate $C$. The candidate is accepted into the $R C L$ if it satisfies $C \geq C_{\min }+\gamma\left(C_{\max }-C_{\min }\right)$. The parameter $\gamma \in[0,1]$ controls the size of the list. If $\gamma=0$, all the blocks go into the list and the selection is completely random. In contrast, $\gamma=1$ means a greedy selection. Only the best candidate goes into the list and therefore is always 

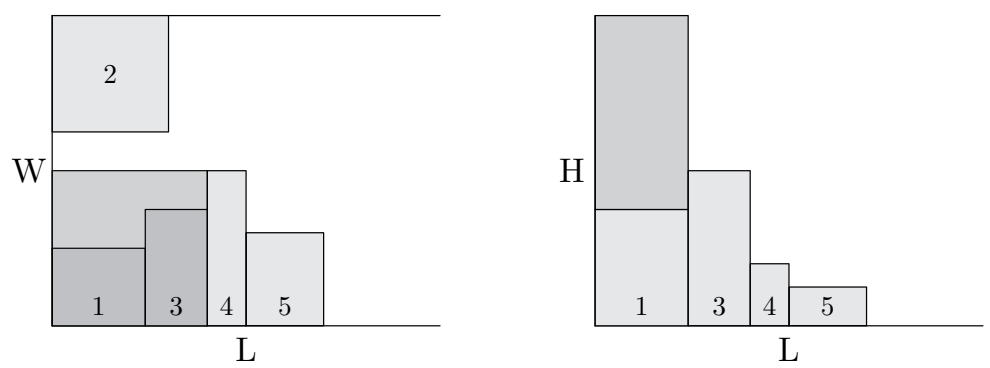

(a) Visible
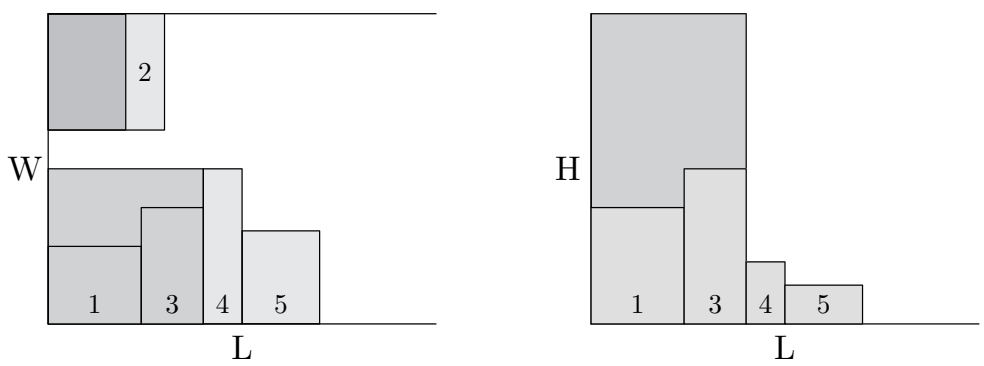

(b) Touchable
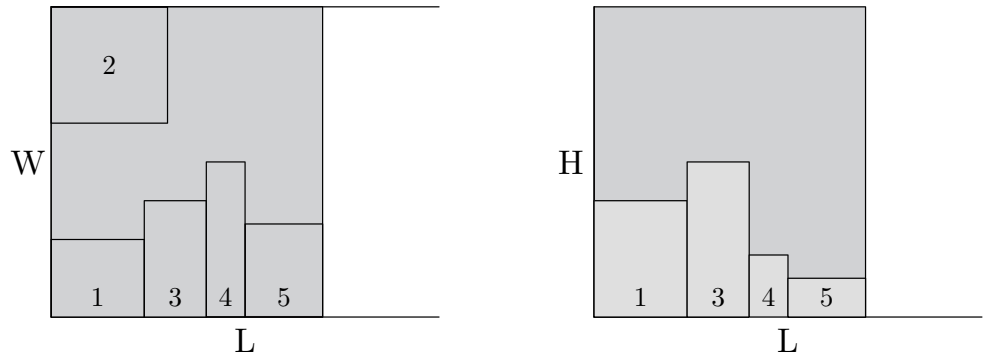

(c) Separated, $\delta=0$

Figure 6 - Updating the maximal spaces after packing the boxes for a client.

chosen. For $0<\gamma<1$, the number of blocks going into the list is not predefined, but it depends on the relative values of the candidates.

We also randomize the criterion used to assign a value to each block. At each iteration, we choose at random between the criterion based on the increase in the occupied volume and the criterion based on the way in which the blocks fit into the space, and build the solution using the criterion chosen.

\subsection{Improvements}

We consider two types of improvements, one of them based on improvements of the global solution and the other consisting of improvements of each client: 
- The first movement consists of eliminating the last $k \%$ boxes packed in the complete solution (for instance, the last 50\%). We choose the value $k$ at random from the interval $[30,90]$. The removed items plus the items that were left unpacked in the solution are then packed again using the deterministic constructive procedure. In this call of the deterministic algorithm we can use the objective function Best-Volume or the objective function Best-Fit. Both alternatives have been tested and their results will appear in the next section. We consider that a solution has improved if the total volume of the packed boxes has increased.

- In the second improvement, we apply the previous procedure to the partial solution we have when we finish packing the boxes for a customer. In this case, we consider that the solution has improved if the area occupied by the partial solution has decreased.

The improvement phase is only called if the solution of the constructive phase is considered to be promising, that is, if it is considered a good starting point for improving on the best known solution. Therefore, we only consider those solutions that are above a certain threshold. At the beginning, the threshold takes the value of the first solution of the constructive algorithm. Then, if at an iteration the solution value is greater than the threshold, we update this threshold to this value and go to the improvement phase. If the solution value is lower than the threshold, the solution is not improved and the reject counter (niter) is increased. When the number of rejected solutions is greater than a value maxFilter, the threshold is decreased according to the expression:

$$
\text { threshold }=\text { threshold }-\lambda(1+\text { threshold })
$$

where $\lambda$ is set at 0.2 and max Filter $=5$, as in Marti \& Moreno [36].

\subsection{Memory-based constructive phase}

When we deal with the unrestrictive variant of the problem we can modify the constructive phase. In this case it is possible not to pack some boxes for a customer or even not to pack all the boxes for one or more customers and continue with the packing for the following customers. This will give us more flexibility which could lead to better solutions.

We save the information relative to the area of the floor of the container used by the partial solution each time the packing of the boxes for a customer is finished. Every Iter $_{m}$ iterations (which we fixed at 500), we compute for each customer the difference between the area used before and after their packing is done and divide it by the total volume of their boxes. The average value of this ratio gives us an estimate of the relative cost of packing the boxes for this customer and we use this information to decide which customers and boxes can be left out of the packing in order to explore possibly better solutions.

For the next Iter $_{r}$ iterations, before starting the loading process, we remove from the list of boxes a percentage of boxes to be packed (Iter $r=\max \{5 *$ customers, 250 $\}$ ). The percentage depends on the quality of the best known solution; we remove at most a random value taken 
from the interval $(0.3 *($ Tot Vol - VolBest $), 0.7 *($ Tot Vol $-V o l B e s t))$, where Tot Vol is the total volume of the boxes to pack and VolBest the volume of the boxes in the best known solution. We take the customer with the greatest ratio and remove boxes randomly until we reach the predefined percentage. If we do not reach the percentage with this customer, we take the customer with the next largest value, and so on. The difference Tot Vol - VolBest indicates the volume of the boxes left unpacked in the best known solution. We want to make room for boxes at the end of the list, eliminating boxes of previous costumers, but ensuring that the reduced list is large enough to fill completely the container.

\section{Computational results}

The algorithm was coded in C++ and run on an Intel Core i7-2600 CPU with $3.40 \mathrm{GHz}$ and $12 \mathrm{~GB}$ of RAM. We used several types of test instances. First, we used the test problems generated by Bischoff \& Ratcliff [5]. The 700 problems are divided into 7 classes $(B R 1, \ldots, B R 7)$ of 100 instances each. The number of box types in each class ranges from 3 different types in the first class to 20 types in the seventh class. This set of 700 instances has been used by many authors and is therefore the most usual benchmark for comparing the algorithms. In these instances the boxes have been generated independently of the container dimensions, so there is no guarantee that all the boxes of one instance will fit into the container. The weight of each box is proportional to its volume and the load-bearing limits have been drawn randomly from a uniform distribution. The problems are available on the ESICUP website: http://paginas.fe.up.pt/ esicup/tikilist_file_gallery.php?galleryId=4. To extend these data sets for the multi-drop problem, Christensen \& Rousoe [12] take a number of customers 2, 5, 10, or 50, and assign each box at random to one of these customers. The assignment is done independently for each number of customers.

We have also used other sets of instances in order to compare with other authors. We compare our algorithm with the exact procedure developed by Junqueira et al. [30] using the 16 instances they generated. We also use the instances by Ceschia \& Schaerf [10] in which they pack a single container. Finally, in order to compare with the procedure by Liu et al. [33], we have generated 100 instances using the information provided in their paper.

For a last comparison with algorithms only considering load-bearing constraints, we have extended the study to classes BR8-BR15 of strongly heterogeneous instances, also developed by Bischoff \& Ratcliff [5]. In these classes, the number of box types ranges from 30 for BR8 to 100 for BR15.

For our GRASP algorithm we have set a maximum number of 50000 iterations. In all the tables the averages correspond to the percentage of volume used.

\subsection{Comparing with Ceschia \& Schaerf [10]}

The first comparison is made with Ceschia \& Schaerf [10]. They consider a container loading problem with orientation, full support, load bearing, and multi-drop constraints, and develop a constructive algorithm that builds walls (as George \& Robinson [23]), using stacks which are 
made of homogeneous blocks of boxes. The boxes are ordered by drop number and the way in which they are packed ensures that the multi-drop constraints are satisfied. A local search improves the solution by changing the sequence of boxes, but respecting the multi-drop conditions.

In their computational results, they use a set of 117 real-world problems, some of them involving just one container and others involving several containers. Here we have used only the 23 instances involving one container. A summary of the results appears in Table 1. The GRASP algorithm matches the reported solutions for 10 instances and improves on the results for the other 13 instances.

Table 1 - Comparison with Ceschia \& Schaerf [10] on instances with one container.

\begin{tabular}{|l|c|c|}
\hline & GRASP & Ceschia \& Schaerf \\
\hline Average volume (\%) & 75,04 & 70,04 \\
\hline Average CPU time (seconds) & 18,66 & 39.41 \\
\hline
\end{tabular}

\subsection{Comparing with Christensen \& Rousoe [12]}

Christensen \& Rousoe [12] considered the version of the multi-drop constraints in which a box can be unloaded if it is visible from the top and from the door of the container. They also included orientation, full support and load-bearing, developed a tree-search algorithm, and tested it on the Bischoff \& Ratcliff [5] instances. Their algorithm was implemented in C++ and the tests were performed on a Linux machine with $2.4 \mathrm{GHz} 64$ bit AMD processor and 2 GB RAM, with a time limit of 60 seconds.

In Table 2 we can see the results of our algorithm compared with the Christensen \& Rousoe [12] algorithm. The table shows that our algorithm obtains better solutions for any number of customers, from the classic problem with just one customer to problems with large numbers of customers, with similar running times. Table 2 also shows that the solution quality depends not only on the number of customers, but also on the combination of the number of box types and the number of customers in the problem. When there are only three box types (BR1) the solution quality only drops $13 \%$ when going from 1 to 50 customers, but in the case of 20 box types (BR7) the difference between 1 and 50 customers is almost 30\%. It is not surprising that the difficult problems have many customers and many box types.

\subsection{Comparing with Liu et al. [33] and with Jin et al. [29]}

Liu et al. [33] considered the home delivery problem in which one box has to be delivered to each costumer, without moving and reloading other boxes. This corresponds to a multi-drop problem in which every client demands just one box. They develop a randomized constructive algorithm and consider two versions: $A 1$, in which the order of clients has to be respected, corresponding to our restrictive case, and $A 2$, in which when a box cannot be packed the process continues with the next boxes until no more boxes can be packed, corresponding to the unrestrictive case. Jin et al. [29] develop a simulated annealing for the general CLP, based on orderings of the boxes to 
Table 2 - Comparison with Christensen \& Rousoe [12].

\begin{tabular}{|c|cccccccc|c|}
\hline \multirow{2}{*}{ Customers } & \multicolumn{7}{|c|}{ GRASP } & Christensen \\
\cline { 2 - 8 } & BR1 & BR2 & BR3 & BR4 & BR5 & BR6 & BR7 & Average & \& Rousoe \\
\hline $\mathbf{1}$ & 92,47 & 92,75 & 92,76 & 92,22 & 91,68 & 91,01 & 89,62 & 91,79 & 89,07 \\
$\mathbf{2}$ & 91,77 & 90,76 & 89,47 & 88,56 & 87,78 & 86,69 & 85,51 & 88,65 & 85,96 \\
$\mathbf{5}$ & 88,56 & 86,02 & 83,15 & 81,36 & 80,14 & 78,30 & 76,12 & 81,95 & 78,52 \\
$\mathbf{1 0}$ & 85,28 & 81,43 & 77,82 & 76,14 & 74,47 & 72,45 & 70,22 & 76,83 & 72,64 \\
$\mathbf{5 0}$ & 79,32 & 73,96 & 68,98 & 66,87 & 64,85 & 63,25 & 60,61 & 68,26 & 64,45 \\
\hline Average & 87,48 & 84,98 & 82,44 & 81,03 & 79,78 & 78,34 & 76,42 & 81,50 & 78,13 \\
\hline Time & 30,29 & 34,01 & 37,93 & 41,98 & 57,18 & 63,35 & 69,94 & 47,81 & 60 \\
\hline
\end{tabular}

be packed. As a particular case, they developed algorithm SIBP for a fixed box ordering. Liu et al. [33] tested the SIBP algorithm and found that it did not guarantee the multi-drop constraints. Then, in order to compare with their algorithms, they used algorithm SIBP', an adapted version of SIBP satisfying the multi-drop constraints. For the tests they used the 100 instances of set BR7 ([5]), adding a random order to the original boxes. Algorithms were run 100000 times on each instance and average results were reported, using an Intel Core 2 Duo E8400 3.00 GHz PC with $2.00 \mathrm{~GB}$ of memory.

We have generated 100 instances following the information provided in their paper, because their original data are unavailable. However, given that their data set has 100 problems and that we report only averages, it is unlikely to introduce any systematic bias in the results.

Table 3 shows that the our GRASP algorithm is able to obtain better results even for this special case of multi-drop constraints in similar computing times.

Table 3 - Comparison with Liu et al. [33] and Jin et al. [29].

\begin{tabular}{|l|ccc|cc|}
\hline & \multicolumn{3}{|c|}{ Restrictive case } & \multicolumn{2}{c|}{ Unrestrictive case } \\
\cline { 2 - 6 } & GRASP & SBIP' & A1 & GRASP & A2 \\
\hline Average & 49,52 & 34.17 & 48,78 & 53.27 & 53.18 \\
\hline Time & 84,11 & 63,76 & 51,98 & 82.14 & 85.68 \\
\hline
\end{tabular}

\subsection{Comparing with Junqueira et al. [30]}

We have also compared our GRASP algorithm with the exact algorithm by Junqueira et al. [30]. We compare with this type of problem for two reasons. First, because they deal with a different type of multi-drop constraint. As explained in Section 3, they separate the boxes for each customer using virtual walls and boxes can only be unloaded if there are less than a given value $\delta$ behind these virtual walls. Second, because they developed an exact model and comparing our results with theirs is a good way of assessing the quality of the solutions obtained by the GRASP algorithm. They did not impose the condition of packing all the boxes for a customer before starting to pack those for the next customer, so they solved the unrestrictive variant of the problem. 
Table 4 - Comparison with Junqueira et al. [30], $\delta=l_{i}$.

\begin{tabular}{|l|c|c|c|c|c|}
\hline \multirow{2}{*}{$\delta=l_{i}$} & Number & Box & Container & \multicolumn{2}{|c|}{ \% Volume packed } \\
\cline { 5 - 6 } & of boxes & types & length & Junqueira et al. & GRASP \\
\hline A1 & 20 & 1 & 15 & 100 & 100 \\
A5 & 41 & 5 & 15 & 100 & 100 \\
& 41 & 5 & 12 & 90 & 100 \\
A10 & 99 & 10 & 15 & 100 & 100 \\
A20 & 89 & 20 & 15 & 100 & 100 \\
B1 & 50 & 1 & 15 & 100 & 100 \\
B5 & 813 & 5 & 15 & 100 & 100 \\
B10 & 1000 & 10 & 15 & 100 & 100 \\
B20 & 674 & 20 & 15 & 100 & 100 \\
\hline
\end{tabular}

Table 5 - Comparison with Junqueira et al. [30], $\delta=0$.

\begin{tabular}{|l|c|c|c|c|c|}
\hline \multirow{2}{*}{$\delta=0$} & Number & Box & Container & \multicolumn{2}{|c|}{ \% Volume packed } \\
\cline { 5 - 6 } & of boxes & types & length & Junqueira et al. & GRASP \\
\hline A1 & 20 & 1 & 15 & 100 & 100 \\
& 20 & 1 & 12 & 95 & 95 \\
A5 & 41 & 5 & 15 & 100 & 100 \\
& 41 & 5 & 12 & 77,9 & 95,5 \\
A10 & 99 & 10 & 15 & 100 & 100 \\
A20 & 89 & 20 & 15 & 100 & 100 \\
& 89 & 20 & 12 & 92,8 & 98,4 \\
B1 & 50 & 1 & 15 & 100 & 100 \\
B5 & 813 & 5 & 15 & 100 & 100 \\
B10 & 1000 & 10 & 15 & 100 & 100 \\
B20 & 674 & 20 & 15 & 100 & 100 \\
\hline
\end{tabular}

They generated a set of instances with different numbers of boxes. The number of customers was set to three for all the problems. Two values of the parameter $\delta$, defining when the boxes are available, were used, $\delta=0$ and $\delta=l_{i}$. Their tests were performed on a PC Core i7 $(2.8 \mathrm{GHz}$, $8.0 \mathrm{~GB}$ ) with a time limit of 3600 seconds.

Tables 4 and 5 show the results obtained considering $\delta=l_{i}$ and $\delta=0$, respectively. The problems in Table 4 , with $\delta=l_{i}$, are less restricted and both algorithms are able to pack all the boxes. When $\delta=0$, in Table 5, the problems are more restricted and in some instances their exact algorithm fails to obtain the optimal solution within the time limit of 3600 seconds. In these cases, our GRASP algorithm is able to obtain equal or better solutions.

\subsection{Problems with only one customer and load bearing constraints}

Although this is not the main purpose of our algorithm, we compare it with the algorithm for the container loading problem with orientation, full support and load bearing constraints, proposed 
Table 6 - Comparing with Alonso et al. [1].

\begin{tabular}{|ccc|ccc|}
\hline Class & GRASP & Alonso et al. & Class & GRASP & Alonso et al. \\
\hline BR1 & $\mathbf{8 2 , 5}$ & 81,4 & BR8 & 83,7 & $\mathbf{8 5 , 5}$ \\
BR2 & $\mathbf{8 6 , 7}$ & 85,7 & BR9 & 82,4 & $\mathbf{8 4 , 8}$ \\
BR3 & $\mathbf{8 8 , 4}$ & 87,3 & BR10 & 81,4 & $\mathbf{8 4 , 0}$ \\
BR4 & $\mathbf{8 8 , 1}$ & 86,9 & BR11 & 80,1 & $\mathbf{8 2 , 8}$ \\
BR5 & $\mathbf{8 7 , 4}$ & 86,6 & BR12 & 78,9 & $\mathbf{8 1 , 3}$ \\
BR6 & $\mathbf{8 6 , 9}$ & 86,3 & BR13 & 77,7 & $\mathbf{8 0 , 2}$ \\
BR7 & 85,53 & $\mathbf{8 5 , 7}$ & BR14 & 76,6 & $\mathbf{7 8 , 9}$ \\
& & & BR15 & 75,5 & $\mathbf{7 8 , 0}$ \\
\hline Average (1-7) & $\mathbf{8 6 , 5}$ & 85,7 & Average (8-15) & $\mathbf{7 9 , 5}$ & 83,7 \\
\hline Time (sec.) & $\mathbf{1 3 , 5}$ & 9,8 & Time (sec.) & $\mathbf{1 0 2 , 8}$ & 64,7 \\
\hline Average(1-15) & 82,8 & $\mathbf{8 3 , 5}$ & \multicolumn{3}{|c}{} \\
\hline
\end{tabular}

by Alonso et al. [1] which has obtained the best results for this type of problem, and use the same test instances. In this case there is only one customer for the complete shipment. They proposed a GRASP algorithm with different the two algorithms can be seen in Table 6. In the first seven classes the new algorithm obtains better results, but for more heterogeneous instances the Alonso et al. [1] algorithm is better. On average, the results obtained by our algorithm can be considered competitive also for instances without multi-drop constraints.

\section{CONCLUSIONS}

We have reviewed different algorithms and types of multi-drop problems. We have developed a GRASP algorithm with a new constructive procedure and some new improvement movements. The main contribution is that this algorithm is able to accommodate the different versions of the multi-drop constraints which have appeared in the literature and it obtains very good results compared with existing heuristics for the different versions of the problem. The algorithm also gets good results for other variants of the problem including full support and load-bearing constraints, even when all the boxes are for the same customer.

The algorithm is flexible and could be adapted to other constraints, such as the maximum weight of the container, weight distribution, and boxes in which the weight is not proportional to the volume. In the near future we plan to use this algorithm to work with more practical situations, such as those combining loading and routing problems.

\section{ACKNOWLEDGEMENTS}

The authors would like to thank Soren Christensen and Leonardo Junqueira, who kindly sent us the sets of instances used in their papers.

This study has been partially supported by the Spanish Ministry of Science and Technology, DPI2011-24977,by Project PPII-2014-011-A, Consejería de Educación y Ciencia, Junta de Comunidades de Castilla-La Mancha, and by Generalitat Valenciana PROMETEO/2013/049. 


\section{REFERENCES}

[1] Alonso MT, Alvarez-Valdes R, Parreño F \& Tamarit JM. 2014. A Reactive GRASP algorithm for the container loading problem with load-bearing constraints. European Journal of Industrial Engineering, 8(5): 669-694.

[2] Araujo OCB \& Armentano VA. 2007. A multi-start random constructive heuristic for the container loading problem. Pesquisa Operacional, 27: 311-331.

[3] Bischoff EE. 2006. Three-dimensional packing of items with limited load bearing strength. European Journal of Operational Research, 168: 952-966.

[4] BISCHOFF EE \& MARRIOTT MD. 1990. A comparative evaluation of heuristics for container loading. European Journal of Operational Research, 44(2): 267-276.

[5] BischofF EE \& RATCLIFF MSW. 1995. Issues in the development of approaches to container loading. Omega, 23: 377-390.

[6] BortFeld A. 2012. A hybrid algorithm for the capacitated vehicle routing problem with threedimensional loading constraints. Computers and Operations Research, 39(9): 2248-2257.

[7] Bortfeldt A \& Gehring H. 2001. A hybrid genetic algorithm for the container loading problem. European Journal of Operational Research, 131: 143-161.

[8] Bortfeldt A, Gehring H \& MACK D. 2003. A parallel tabu search algorithm for solving the container loading problem. Parallel Computing, 29(5): 641-662.

[9] Bortfeldt A \& W ÄScher G. 2013. Constraints in container loading - A state-of-the-art review. European Journal of Operational Research, 229(1): 1-20.

[10] Ceschia S \& Schaerf A. 2013. Local search for a multi-drop multi-container loading problem. Journal of Heuristics, 19: 275-294.

[11] Ceschia S, Schaerf A \& StÜtZle T. 2013. Local search techniques for a routing-packing problem. Computers and Industrial Engineering, 66(4): 1138-1149.

[12] Christensen SG \& Rousoe DM. 2009. Container loading with multidrop constraints. International Transactions in Operational Research, 16(6): 727-743.

[13] Doerner KF, Fuellerer G, Gronalt M, Hartl RF \& Iori M. 2007. Metaheuristics for the vehicle routing problem with loading constraints. Networks, 49: 294-307.

[14] Egeblad J, Garavelli C, Lisi S \& Pisinger D. 2010. Heuristics for container loading of furniture. European Journal of Operational Research, 200(3): 881-892.

[15] ELEY M. 2002. Solving container loading problems by block arrangement. European Journal of Operational Research, 141: 393-409.

[16] Feo T \& Resende MGC. 1989. A Probabilistic Heuristic for a Computationally Difficult Set Covering Problem. Operations Research Letters, 8: 67-71.

[17] FAnslau T \& BortFeldT A. 2010. A tree search algorithm for solving the container loading problem. INFORMS Journal of Computing, 22: 222-235.

[18] FeKete SP, SChepers J \& VAN DER VEen JC. 2007. An exact algorithm for higher-dimensional orthogonal packing. Operations Research, 55: 569-587.

[19] GeHRING H \& BORTFELDT A. 1997. A genetic algorithm for solving the container loading problem. International Transactions in Operational Research, 4(5-6): 401-418. 
[20] Gehring H \& BortFeldt A. 2002. A parallel genetic algorithm for solving the container loading problem. International Transactions in Operational Research, 9: 497-511.

[21] Gendreau M, Iori M, Laporte G \& Martello S. 2006. A tabu search algorithm for a routing and container loading problem. Transportation Science, 40(3): 342-350.

[22] Gendreau M, Iori M, Laporte G \& Martello S. 2008. A Tabu search heuristic for the Vehicle Routing Problem with two-dimensional loading constraints. Networks, 51(1): 4-18.

[23] George JA \& Robinson DF. 1980. A heuristic for packing boxes into a container. Computers and Operations Research, 7(3): 147-156.

[24] Gilmore PC \& Gomory RE. 1965. Multistage cutting stock problems of two and more dimensions. Operations Research, 13: 94-120.

[25] HAessler RW \& TALBOT FB. 1990. Load planning for shipments of low density products. European Journal of Operational Research, 44(2): 289-299.

[26] HIFI M. 2002. Approximate algorithms for the container loading problem. International Transactions in Operational Research, 9(6): 747-774.

[27] Iori M, Salazar JJ \& Vigo D. 2007. An Exact Approach for the Vehicle Routing Problem with Two-Dimensional Loading Constraints. Transportation Science, 41: 253-264.

[28] IORI M \& MARTELlo S. 2010. Routing problems with loading constraints. TOP, 18: 4-27.

[29] Jin Z, OHNo K \& DU J. 2004. An efficient approach for the three-dimensional container packing problem with practical constraints. Asia-Pacific Journal of Operational Research, 21(3): 279-295.

[30] Junqueira L, Morabito R \& Yamashita DS. 2012. MIP-based approaches for the container loading problem with multi-drop constraints. Annals of Operations Research, 199(1): 51-75.

[31] Junqueira L, Morabito R \& Yamashita DS. 2012. Three-dimensional container loading models with cargo stability and load bearing constraints. Computers and Operations Research, 39: 74-85.

[32] LAI KK, XUE J \& XU B. 1998. Container packing in a multi-customer delivering operation. Computers and Industrial Engineering, 35(1-2): 323-326.

[33] LIU WY, LIN CC \& Y U CS. 2011. On the three-dimensional container packing problem under home delivery service. Asia-Pacific Journal of Operational Research, 28(5): 601-621.

[34] Mack D, Bortfeldt A \& Gehring H. 2004. A parallel hybrid local search algorithm for the container loading problem. International Transactions in Operational Research, 11(5): 511-533.

[35] Martello S, Pisinger D \& Vigo D. 2000. The three-dimensional bin packing problem. Operations Research, 48(2): 256-267.

[36] Marti R \& Moreno-Vega JM. 2003. Metodos multiarranque. Inteligencia Artificial, 19(2): 49-60.

[37] Morabito R\& Arenales M. 1994. An AND/OR-graph approach to the container loading problem. International Transactions in Operational Research, 1(1): 59-73.

[38] Moura A \& Oliveira JF. 2005. A GRASP approach to the container loading problem. IEEE Intelligent Systems, 20: 50-57.

[39] NGOI BKA, TAY ML \& CHUA ES. 1994. Applying spatial representation techniques to the container packing problem. International Journal of Production Research, 32: 111-123. 
[40] Parreño F, Alvarez-Valdes R, Oliveira JF \& Tamarit JM. 2008. A Maximal-Space Algorithm for the Container Loading Problem. INFORMS Journal on Computing, 20(3): 412-422.

[41] Parreño F, Alvarez-Valdes R, Oliveira JF \& TAmarit JM. 2010. Neighborhood structures for the container loading problem: A VNS implementation. Journal of Heuristics, 16: 1-22.

[42] Parreño F, Alvarez-Valdes R, Oliveira JF \& Tamarit JM. 2010. A hybrid GRASP/VND algorithm for two- and three-dimensional bin packing. Annals of Operations Research, 179(1): 203-220.

[43] PISINGER D. 2002. Heuristics for the container loading problem. European Journal of Operational Research, 141(2): 382-392.

[44] RATCLIFF MSW \& BISCHOFF EE. 1998. Allowing for weight considerations in container loading. OR Spectrum, 20: 65-71.

[45] REN J, TIAN Y \& SAWARAgi T. 2011. A tree search method for the container loading problem with shipment priority. European Journal of Operational Research, 214(3): 526-535.

[46] Resende MGC \& Ribeiro CC. 2003. Greedy Randomized Adaptive Search Procedures. In: Glover F \& Kochenberger G (editors), Handbook of Metaheuristics, pages 219-249, Kluwer, Boston.

[47] Terno J, Scheithauer G, Sommerweiss \& Riehme J. 2000. An efficient approach for the multi-pallet loading problem. European Journal of Operational Research, 123: 372-381.

[48] Wëscher G, HaUsSner H \& SChUmann H. 2007. An improved typology of cutting and packing problems. European Journal of Operational Research, 183(3): 1109-1130.

[49] ZHU W, QIN H, LIM A \& WANG L. 2012. A two-stage tabu search algorithm with enhanced packing heuristics for the 3L-CVRP and M3L-CVRP. Computers and Operations Research, 39(9): 21782195 . 